\section{Außerschulische Situation und Freizeit von einzelinkludiert beschulten Jugendlichen mit Hörschädigung in der Sekundarstufe an allgemeinen Schulen}

\section{Sabine Rixen \\ Ludwig-Maximilians-Universität München}

\section{Forschungshintergrund}

Das Jugendalter ist durch Umbrüche und eine Vielzahl komplexer Entwicklungsaufgaben gekennzeichnet (Hurrelmann \& Quenzel, 2016). Dazu gehört beispielsweise einerseits, den sich verändernden Körper zu akzeptieren sowie eine stabile und autonome Ich-Struktur aufzubauen, aber andererseits auch, sich auf geänderte Lernbedingungen (Fachlehrerprinzip, häufige Raumwechsel usw.) und sich ändernde Verhaltensmuster unter Gleichaltrigen einzustellen.

Als Teil des Forschungsprogramms zur schulischen Integration und Inklusion von Kindern und Jugendlichen mit einer Hörschädigung an allgemeinen Einrichtungen, das unter Leitung von Prof. Annette Leonhardt seit 1999 zahlreiche Teilaspekte im inklusiven Setting beleuchtet, baut dieses Projekt auf Ergebnissen der vorangegangenen Module auf und erfasst weitere Aspekte im außerschulischen Kontext. Hierbei handelt es sich nicht ausschließlich um ein Forschungsthema, das im Bereich der Freizeitforschung (Opaschowski, 2008) verortet ist, vielmehr bezieht es Aspekte schulischer Inklusion mit ein, die einen Einfluss auf das Freizeitverhalten und Freizeiterleben junger Menschen mit Hörschädigung haben. Ein Bereich, der bisher eher randständig betrachtet wurde.

Wichtige inhaltliche Impulse ergaben sich unter anderem aus dem zum Forschungsprogramm gehörenden Forschungsprojekt ,Die soziale Situation integriert beschulter Kinder und Jugendlicher mit Hörschädigung an der allgemeinen Schule' (Gräfen, 2014) und erweitert die Thematik um die außerschulische Situation. Spannende Querverbindungen ergeben sich auch mit dem aktuell laufenden Forschungsprojekt von D. Eck, das die Gelingensbedingungen von Inklusion mit Hörschädigung in der Sekundarstufe erfasst (Eck, 2018).

\section{Forschungsziel und Forschungsfragen}

Geleitet wird das Forschungsprojekt von der zentralen Forschungsfrage:

- Wie gestalten und erleben Jugendliche mit Hörschädigung (etwa ab dem Alter von 12 Jahren) den außerschulischen Bereich?

Konkretisiert wird diese Frage von folgenden Unterfragen:

Wie erleben und beschreiben Eltern die außerschulische Situation ihres Kindes?

n In welchem Verhältnis stehen Obligationszeit und Dispositionszeit?

- Wie gestalten sich soziale Beziehungen?

- Welche Barrieren werden bei der schulischen Inklusion von Jugendlichen mit einer Hörschädigung erkennbar und wie wirken sich diese auf ihr Freizeitverhalten aus?

- Werden Ressourcen erkennbar, die für die Bewältigung des Alltags relevant sind?

\section{Methodisches Vorgehen}

Durchgeführt wird das Forschungsprojekt als multimethodische Studie.

In der ersten Phase, einer qualitativen Erhebung mittels leitfadenorientierten Interviews, wird das subjektive Freizeiterleben der Jugendlichen erfasst und näher beschrieben. Darauf folgt in der zweiten Phase eine quantitative Erhebung mittels Fragebogen.

In diesem bislang wenig betrachteten Bereich ist es wichtig, zunächst die subjektive Sicht der Betroffenen zu erfassen und dabei Haltungen, Erlebnisse und Einstellungen zu erfahren. Da Jugendliche mit einer Hörschädigung als Experten für ihre Situation gesehen werden, sollen sie selbst zu Wort kommen. Ein Erhebungsinstrument, das einerseits durch ein Grundgerüst an Fragen Struktur gibt, aber andererseits durch größtmögliche Offenheit und narrative Impulse Möglichkeiten für einen individuellen Fokus eröffnet, scheint in hohem Maße dazu geeignet, subjektives Erleben zu erfassen (vgl. Reinders, 2016).

Die Auswahl der Probanden der Interviewstudie erfolgte gezielt danach, dass versucht wurde, in der qualitativen Phase ein breites Spektrum an 
vorab festgelegten Kriterien abzudecken (Hörstatus, Schulart, Alter, Geschlecht), um möglichst unterschiedliche Erkenntnisse zu gewinnen und um eine breite Basis für den quantitativen Teil der Studie zu bekommen.

Befragt wurden drei Jugendliche $(\mathrm{w} / \mathrm{m})$ im Alter von 12, 15 und 18 Jahren, die mit einer mittelgradigen Hörschädigung, einer mittel-hochgradigen Hörschädigung und Gehörlosigkeit eine Realschule, ein Gymnasium und eine Fachoberschule besuchen. Alle Jugendlichen sind mit Hörtechnik (HG bzw. Cl) versorgt, die konsequent getragen wird, zusätzliche Übertragungstechnik wird nur in einem Fall eingesetzt. Ein dazu gehörendes Elternteil, mit einem eigenen Elternleitfaden befragt, trianguliert die Perspektive der Jugendlichen, integriert weitere Aspekte und zeigt gegebenenfalls Parallelen zu einem Geschwisterkind auf.

Grundlage zur Entwicklung des Fragebogens für den quantitativen Teil der Forschungsarbeit werden die Ergebnisse der beschriebenen Interviewstudie sein, in die auch Erkenntnisse aus Interviews mit Jugendlichen einfließen, die im Rahmen von Bachelorarbeiten entstanden. Die quantitative Erhebung mittels Fragebogen soll in Gesamtbayern durchgeführt werden.

Aktuell befinden sich die Interviews in der Auswertung. Tonaufnahmen wurden angefertigt und transkribiert, und derzeit erfolgt eine computergestützte (MAXQDA) qualitative Inhaltsanalyse (Mayring, 2015).

\section{Erste Erkenntnisse}

Aus den bisher geführten Interviews ergibt sich, dass ein Teil der Jugendlichen mit Hörschädigung auch im außerschulischen Bereich erheblich durch das schulische Setting belastet ist und sich die Belastung vielschichtig äußert. Dass die Jugendlichen zum Teil sehr viel Zeit für Arbeiten mit schulbezogenen Inhalten aufwenden (Hausaufgaben, Nacharbeiten, Lernen usw.), diese Zeit aber nicht mit dem Grad der Hörschädigung korreliert, sondern erheblich von weiteren Faktoren beeinflusst wird. Als förderliche oder hinderliche Faktoren lassen sich eigene kommunikative Strategien erkennen sowie die Akzeptanz der Hörschädigung. Nicht unerhebliche Auswirkungen auf den nachschulischen Bereich scheint die Haltung der unterrichtenden Lehrkräfte sowie der Mitschülerinnen und Mitschüler zu haben, aber auch Vorerfahrungen aus der Grundschulzeit spielen eine Rolle.

Es ist anzunehmen, dass familiäre Gewohnheiten einen gewissen Einfluss auf die Gestaltung der Peerkontakte haben, sie scheinen aber nicht entscheidend für Qualität und Umfang außerfamiliärer Sozialkontakte und Beziehungen zu sein.

Weitere Faktoren wurden aktuell identifiziert und werden im nächsten Forschungsabschnitt näher beleuchtet.

\section{Ausblick}

Eine Triangulation der Ergebnisse aus der qualitativen und der quantitativen Erhebung ermöglicht es, der Vielschichtigkeit der Thematik gerecht zu werden und einzelne Faktoren genauer zu untersuchen.

Weitere interessante Aspekte, die in der quantitativen Erhebung näher beleuchtet werden sollen, sind exemplarisch genannt der Einfluss von Unterstützern aus dem Peerbereich, Resilienzfaktoren oder individuelles Belastungserleben bei individuell vergleichbaren zeitlichen Belastungssituationen.

Weitere Informationen und Literaturangaben können gerne eingeholt werden bei s.rixen@edu.Imu.de 